\title{
Real-time Signal Processing for Shipborne FOG Based on Lifting Wavelet Analysis
}

\author{
Bin $\mathrm{Yi}^{1, \mathrm{a}}$, Qiliang Bao ${ }^{2, \mathrm{~b}}$ \\ ${ }^{1}$ Key Laboratory of Optical Engineering, Chinese Academy of Sciences, Chengdu 610209, China; \\ ${ }^{2}$ Key Laboratory of Optical Engineering, Chinese Academy of Sciences, Chengdu 610209, China; \\ ayibin123457@163.com, bcontrol@ioe.ac.cn
}

Keywords: Lifting wavelet, shipborne FOG, real-time signal processing.

\begin{abstract}
In the visual axis stabilization system under the background of the shipboard, it is necessary to use the high-frequency and low-frequency of the FOG output signal, respectively. For the practical application, this paper proposes a method which contains de-noising and signal separation. This paper analysis the characteristics of the FOG output signal under shipboard conditions, and then based on lifting wavelet transform, select the appropriate wavelet basis, appropriate sliding window size and decomposition scale. Use MATLAB software simulation, the results show that the algorithm can meet realization of real-time premise de-noising and signal separation.
\end{abstract}

\section{Introduction}

Fiber optic gyroscope (FOG) is a new optical gyro based on the Sagnac effect, compared with the traditional mechanical gyroscopes; it is widely used in various fields for its light weight, small size, short start-up time, good reliability, long life, resistance to shock and vibration, large bandwidth, etc.

In the visual axis stabilization system under the background of the shipboard, the main role of FOG is detecting the change of the ship's posture, high-frequency vibration and wind resistance. The detected signal provides a control signal for the stable feedforward control section; providing a guarantee for the final visual axis stabilization system. In this context, we use FOG to high precision measure the signal, at the same time we want to separate the measured signal into a low frequency signal and high frequency signal, respectively as a coarse stabilization, accuracy stabilization control signal.

Wavelet transform is a time-frequency localization analysis method, its window size is fixed but shape is variable, the time window and frequency window can be changed [1]. That is to say it has a low time resolution and a high frequency resolution at low frequencies with high time resolution and low frequency resolution at high frequency portion. It is very suitable for non-stationary signal analysis and feature extraction, known as "mathematics microscope".

The literature [2] proposes a combination of hard threshold, forced de-noising and with sliding window in real time noise reduction program, which is based on lifting wavelet algorithm, realize the real-time signal de-noising of FOG, its computing speed is fast, the effect is obvious. Based on the analysis of the output signal from the shipborne FOG, we gives an improved real-time wavelet analysis algorithm, which can achieve de-noising and separation high and low frequency signals in real-time systems.

\section{Output Signal Analysis of Shipborne FOG}

Because of waves and other disturbances, the movement of ship may be seen as a series of sine wave signals of similar amplitude and frequency combination [3]. In this paper, the background of shipboard conditions, we first analyze the characteristics of FOG output signal; figure 1 is the measured output signal and spectrum analysis of FOG under certain shipboard conditions. 


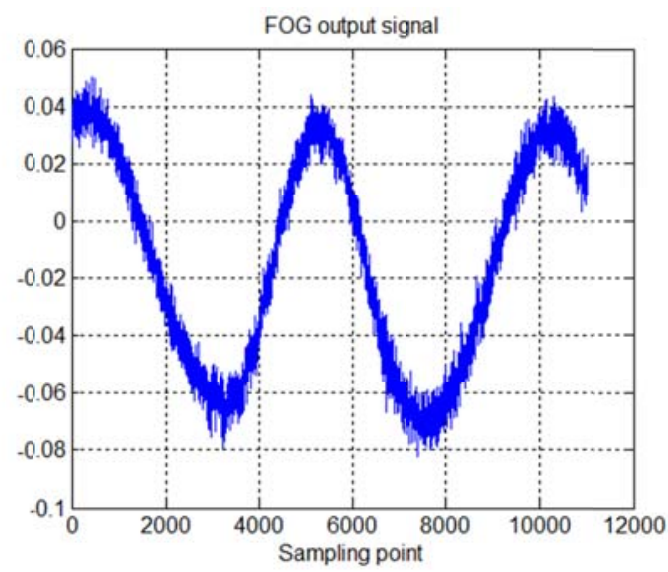

(a) Output signal
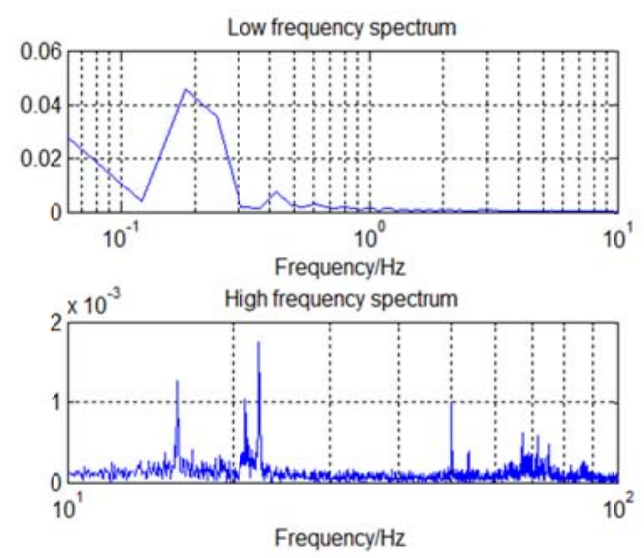

(b) Signal spectrum curve

Fig. 1 Shipborne FOG output signal and spectrum curve

From the figures above show that the FOG output signal under this background includes three distinct frequency segments, they are less than $1 \mathrm{~Hz}$ low frequency signal, around $20 \mathrm{~Hz}$ and $60 \mathrm{~Hz}$ high frequency signal. Where the low frequency signal below $1 \mathrm{~Hz}$ is caused by large swings in the hull, in the five sea conditions, orientation perturbation is mainly about $0.2 \mathrm{~Hz}$, pitch perturbation is mainly about $0.1 \mathrm{~Hz}$, but the perturbation amplitude is great, the maximum angular velocity perturbations of azimuth and elevation are $20.5^{\circ} / \mathrm{s}$ and $12.53^{\circ} / \mathrm{s}$, respectively [4]. Through the analysis we can know, $20 \mathrm{~Hz}$ near the high-frequency signal is caused by the rotation of the propeller of the ship, $60 \mathrm{~Hz}$ near is the multiple of the propeller rotation frequency.

\section{Lifting Wavelet Analysis}

Traditional wavelet transform contains a large number of convolution operations and the operation time is long, efficiency is low, so it is difficult to obtain applications in practical engineering. In 1998, scholars Daubechies I and Sweldens W put forward a new wavelet transform; it does not depend on the Fourier transform, called the lifting wavelet transform format. Lifting wavelet transform signal processing directly in the time domain, changing the traditional wavelet transform the signal into the frequency domain, it has a simple structure, low computation, site operations, saves storage space, the inverse transform can achieve direct reversal and other fine features, greatly reduces the time of arithmetic operations to meet the real-time and application requirements for embedded devices. Lifting wavelet transform is divided into split, predict and update three steps [5].

a. Split

Generally divide the signal $x[n]$ into two sub-sequences, which are odd and even sequence.

$$
\left\{\begin{array}{l}
c_{l}^{0}=x_{2 l} \\
d_{l}^{0}=x_{2 l+1}
\end{array} \quad l=0,1,2, \cdots, \frac{n}{2}-1\right.
$$

b. predict

To make consistent with the simulation software, we use the odd sequence to predict even sequence, and obtain the predicted error as wavelet coefficients, the $i$-th promotion process as follows:

$$
c_{l}^{i}=c_{l}^{i-1}-P\left(d_{l}^{i-1}\right) \quad l=0,1,2, \cdots, \frac{n}{2} ; i=1,2,3 \cdots, m
$$

Where, $P(\cdot)$ is the prediction operator, $i$ represents the $i$-th lifting process.

c. update

Use wavelet coefficients and update operator $U(\cdot)$ to update odd sequence, the result are the scale coefficients. Update is also considered to smoothing of the signal by the filter. 


$$
d_{l}^{i}=d_{l}^{i-1}-U\left(c_{l}^{i}\right) \quad l=0,1,2, \cdots, \frac{n}{2} ; i=1,2,3 \cdots, m
$$

Where, $U(\cdot)$ is the update operator, $i$ represents the $i$-th lifting process.

Through the above three steps completed one lifting process, a wavelet transform needs many times lifting processes to achieve. From this process, we can see that this algorithm can achieve the site of operation, subordinate lifting conversion process only need part of the output of the superior lifting conversion process, in each bit can use the new data to replace the old data. Figure 2 is a schematic diagram of the lifting wavelet transform and inverse transform. In the figure above as long as the corresponding opposite sign can be, because the actual symbol is the result of the above figure and symbol symbolic yard operator controlled together. Lifting wavelet transform inverse transform is the inverse of the above three steps, not enumerate here.

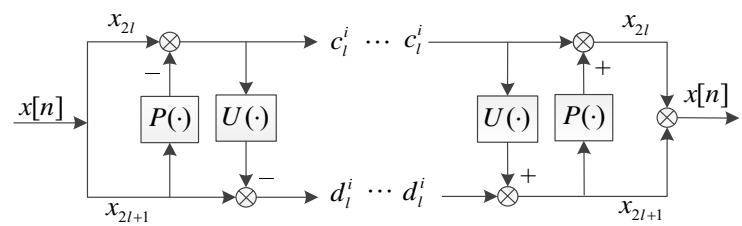

Fig. 2 Schematic diagram of the lifting wavelet transform and inverse transform

\section{FOG Wavelet Real-time Processing Program}

Traditional wavelet analysis algorithm used in off-line processing, in order to meet real-time processing for the FOG collection data, we need to use a sliding window method to intercept data. Its working principle is: First determine the width of the sliding window, then if the number of data points sampled is less than window width remains constant, direct output. When the sampling points is equal to the width of the window, process data using lifting wavelet transform within the data window, the processing result of the last point of this time is the real-time output, while the window is moved forward one unit of time to receive the next sample value, discard the first sample value in the window, and so the cycle to achieve real-time signal processing.

And in order to solve the boundary effect of wavelet transform, the signal needs to be extension. After analysis the FOG output signal characteristic we choose to use symmetric extension.

Algorithm flow chart shown in figure 3:

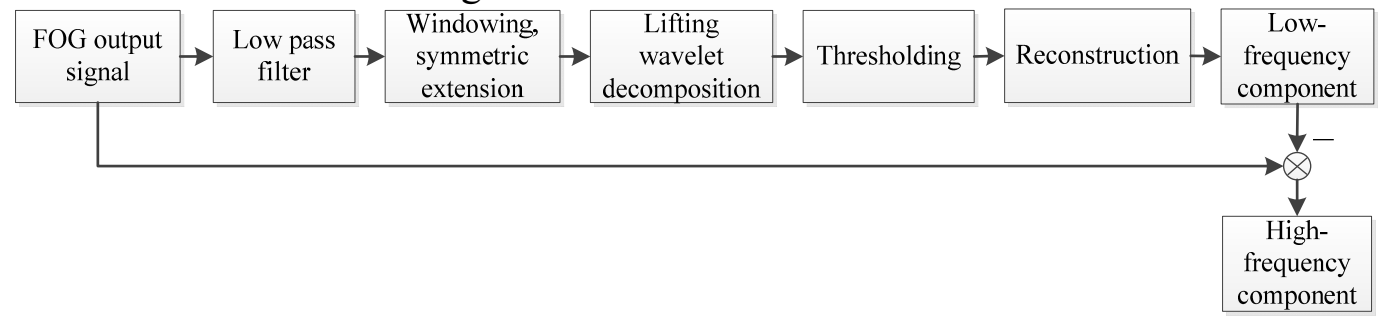

Fig. 3 Algorithm flow chart

Specific procedures are as follows:

1) FOG output signal through a low pass filter which cut-off frequency is $100 \mathrm{~Hz}$;

2) The filtered signal windowing process, the interception of the window size of $\mathrm{N}$ data segments, and then the symmetric extension processing;

3) reconstruction, output the low frequency signal components;

4) Subtracting the low frequency signal components of the filtered signal, then output the signal of the high-frequency component;

5) Discard the first data point within the window, the rest of the data points shifted left, input the next time data point after filtering;

Repeat steps 2-5, you can achieve real-time processing algorithms. Low pass filter selection Chebyshev Type I IIR low-pass filter, and set the cut-off frequency is $100 \mathrm{~Hz}$, attenuation is $80 \mathrm{db}$, filter order is 16 .

The selection of wavelet function not only affects the signal separation effect, it will directly affect the speed of operation [6]. In theory, the scale of the wavelet decomposition is larger, the 
greater the degree of separation of high and low-frequency, but the larger the amount of calculation, the longer time needed, the greater the delay, in the case of high dynamic real-time is worse. Therefore, combine the frequency spectrum analysis of the FOG output signal, we chose db4 wavelet, decomposition scale is 7 , and the width of sliding window is 128 . With db4 wavelet for example, the lifting scheme achieves as following:

$$
\left\{\begin{array}{l}
c_{l}^{0}=x_{2 l} \\
d_{l}^{0}=x_{2 l+1} \\
c_{l}^{1}=c_{l}^{0}-0.3223 c_{l+1}^{0} \\
d_{l}^{1}=d_{l}^{0}-1.1171 c_{l}^{1}-0.3001 c_{l-1}^{1} \\
c_{l}^{2}=c_{l}^{1}-0.0188 d_{l+2}^{1}+0.1176 d_{l+1}^{1} \\
d_{l}^{2}=d_{l}^{1}+2.1318 c_{l}^{1}+0.6364 c_{l-1}^{1} \\
c_{l}^{3}=c_{l}^{2}-0.4691 d_{l}^{2}+0.1400 d_{l-1}^{2}-0.0248 d_{l-2}^{2} \\
d_{l}=0.7341 d_{l}^{2} \\
c_{l}=1.3622 c_{l}
\end{array}\right.
$$

Approximate treatment all the coefficients, so that they are available through a shift-and-add operation to achieve [7]. Such as $-0.3223 \approx-\left(\frac{1}{4}+\frac{1}{16}+\frac{1}{128}+\frac{1}{512}\right)$, thus the allgorithm involves only integer addition, multiplication and shift operations, will make the operation speed greatly accelerated.

\section{Results and Discussion}

We use a fiber optic gyroscope output data in a shipborne experiment to verify the real-time filtering and decomposition algorithm in this paper. The width of the sample is 11,000 points, the sampling frequency is $1000 \mathrm{~Hz}$, and the original data is given in the second part. Using MATLAB software simulation this algorithm, first through a low-pass filter which the cutoff frequency is $100 \mathrm{~Hz}$, and then separated the signal, select db4 wavelet, window size is 128 , decomposition level is 7 layers. Low-frequency signal component and a high-frequency signal component separated as shown in figure 4.
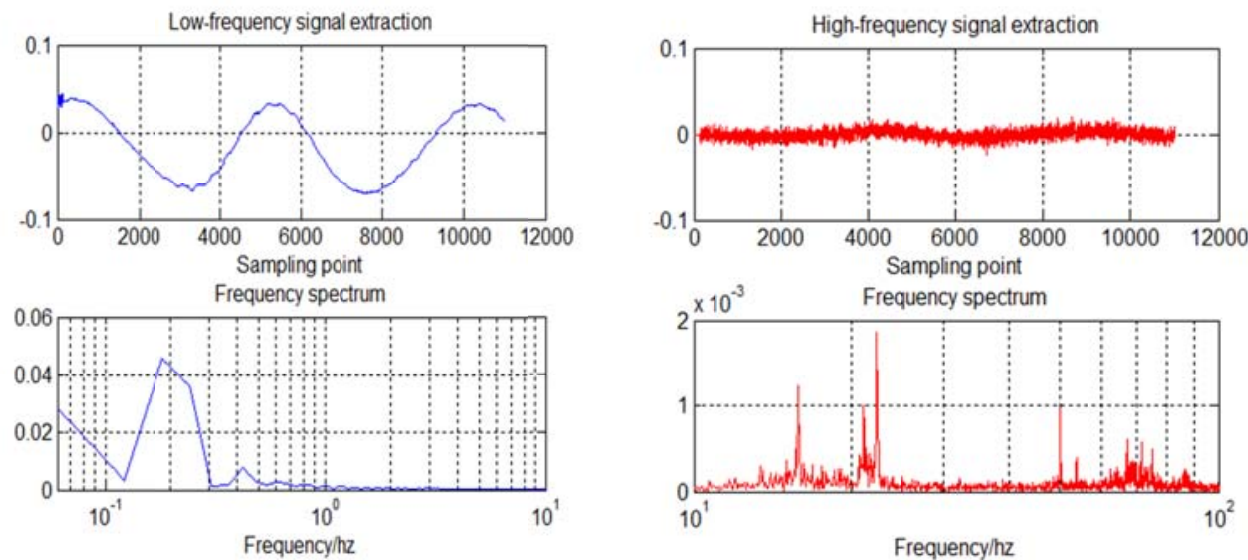

Fig. 4 Low frequency and high frequency signal component isolated

As can be seen from the figure above, the high-frequency contains some of the low-frequency, which is due to the wavelet transform has certain lag.

The figure shows that after processing the FOG output data using the design algorithms, we can effectively suppress noise in the signal, while completing the high and low frequency signal separation. 


\section{Conclusion}

In this paper, we use lifting wavelet analysis method to construct a real-time filtering and separation algorithm, using MATLAB and measured data carried out simulation. The results show that this method can meet real-time while effectively suppressing noise of the output signal and the achievement of high and low frequency signal separation, provides a more reliable control signal for the visual axis stabilization system.

\section{References}

[1]. Cheng Jianhua, Li Mingyue, Rong Wenting, et al. Research and Realization of Real-time Wavelet Filter Algorithm for Shipborne FOG, 2011 IEEE International Conference on Mechatronics and Automation. Beijing, China, Aug. 7-10, p.1872-1876.

[2]. Gao Wei, Zu Yue, Wang Wei. Research on real-time de-noising of FOG based on second generation wavelet transform. Chinese Journal of Scientific Instrument. Vol. 33 (2012) No. 4, p.774-780.

[3]. Han Junhai, Chen Jiabin. A Study on the Rapid Transfer Alignment in Marine Environment. Journal of Beijing Institute of Technology. Vol. 24 (2004) No. 2, p.896-897.

[4]. Xia Yunxia. Study on Control Techniques of Inertial Stabilization on Moving Platform. (Doctor-rate, UCAS, China, 2013). p. 59-59.

[5]. X. Song, C. Zhou, D.M. Hepburn, G. Zhang, Second generation wavelet transform for data de-noising in PD measurement, IEEE Transactions on Dielectrics and Electrical Insulation Vol. 14 No. 6 (2007), p. 1531-1537.

[6]. Jiang Xingguo, Bao Qiliang, Yang Wenshu, et al. Real-time Processing Method Based on Wavelet for Signals of Inertial Sensors. Opto-Electronic Engineering. Vol. 35 (2008) No. 2, p. 12-15.

[7]. Luo Lei, Li Yuehua, Luan Yinghong. Real-Time De-nosing of MMW Detector Signal Based on Lifting Scheme 9- 7Wavelet. Electronics Optics \& Control. Vol17 (2010) No. 2, p74-77. 